\title{
Management of Post-operative LASIK Patients Before and After COVID-19: New Treatment Protocols
}

\section{Karl Stonecipher ${ }^{1 *}$, Ben Likins ${ }^{4}$, Maya Goyal ${ }^{3}$, Monica Soni ${ }^{1}$ and Madison Thurmond ${ }^{2}$}

${ }^{1}$ Department of Clinical Associate Professor of Ophthalmology, University of North Carolina, USA

${ }^{2}$ Department of Biology, University of North Carolina at Wilmington, USA

${ }^{3}$ Georgetown University, USA

${ }^{4}$ Medical University of South Carolina, USA

*Corresponding Author: Karl Stonecipher, Department of Clinical Associate

Professor of Ophthalmology, University of North Carolina, USA.
Received: October 04, 2021

Published: October 23, 2021

(C) All rights are reserved by Karl

Stonecipher., et al.

\begin{abstract}
Importance: To discuss a new post-operative (post-op) protocol for patients undergoing laser-assisted in situ keratomileusis (LASIK) seen during the COVID-19 pandemic.

Background: With advances in analytic software for topography-guided LASIK, better visual outcomes and lower rates of post-op complication and the need for enhancement surgery have allowed for safe yet effective ways to manage patients before, during, and after surgery. However, with the present state of practicing in a pandemic the authors propose a new practice pattern to promote efficiency, maintain outcomes, and potentially reduce ophthalmic complications while keeping our patients safe.

Design: A insignificant risk retrospective review of topography-guided LASIK using two separate protocols. The authors used one prior to the pandemic and another during the pandemic with new patient safety guidelines. Comparison of outcomes will be discussed.

Participants: A total of 1,026 eyes from 513 patients were treated with LASIK prior to the pandemic and during the time of COVID-19 were included in the patient population.

Methods: Management of patients with current post- op protocol including in-patient visits at preoperative, one day, one week, one month, three months and twelve months compared with a new protocol including in-patient visits at preoperative, post op one day, three months and twelve months but telehealth visits with subjective questionnaire at one week and one month were utilized during the pandemic as well as patient isolation from other patients were also instituted preoperatively and postoperatively during the pandemic.
\end{abstract}

Main Outcome Measures: Results included uncorrected visual acuity (UCVA) outcomes for patients in each group as well as total enhancement rates.

Results: No significant differences were seen between UCVA outcomes between groups. The rate of enhancement was also insignificant. Postoperative day 1 UCVA 20/20 or better in individual eyes (OD/OS) was 97\% vs 99\% in Pre-COVID-19 vs Post-COVID-19 
respectively. Postoperative day 1 UCVA $20 / 15$ or better in both eyes (OU) was $81 \%$ vs $84 \%$. The enhancement rate at one year in each group was identical at $0.29 \%$.

Conclusion: The application of advanced analytic computer software engine allows for better planning of LASIK and provides an opportunity for a more efficient and safe protocol for management of patients. Reduction in overall diagnostics and the use of telemedicine also provided benefits to patients and similar outcomes, as compared to prior to the pandemic. No patients during the treatment period developed COVID-19 to our knowledge.

Keywords: Laser-assisted In Situ Keratomileusis (LASIK); COVID-19; Telehealth; Telemedicine; Post-operative (post-op) Management; Uncorrected Visual Acuity (UCVA)

\section{Introduction}

With over 700,000 LASIK surgeries performed in the United States in 2020, it is one of the most popular elective procedures done each year [1]. This number is expected to increase with many people working from home on the computer, becoming vaccinated, practicing social distancing, and wearing mask during the recent COVID-19 pandemic. During this time, advances are being made to provide the safest and highest quality of laser surgery for our patients while also reducing exposure of our patients to other patients and staff to patients.

Two types of corneal refractive procedures that have been widely adapted for clinical use are composed of wavefront optimized (WFO) and topography guided treatments [2,3]. With WFO treatments, the ability to link measured wavefront aberrations in the human eye to contrast sensitivity and visual symptoms enable surgeons to create laser corneal profiles to treat refractive errors [4]. This algorithm was followed using corneal topography to create ablation profiles in the attempt to better predict changes on the anterior surface of an individual's eye. A prospective FDA study showed that excellent vision outcomes occurred when using Contoura ${ }^{\circledR}$ Vision, a topography-guided software, which found a UDVA of 20/20 or better in $92.6 \%$ of participants at one year [2]. The results were corroborated in a study done by Stonecipher., et al. that showed more patients achieved better than 20/20 vision with Contoura ${ }^{\circledR}$ Vision than with WFO: $81.0 \%$ vs $73.0 \%$ respectively [5]. While both types of LASIK treatments provide excellent visual outcomes, the use of topography-guided laser correction may provide better visual outcomes with more patients achieving 20/15 or better.
One of the main limitations of the FDA study showing the potential use of topography-guided laser surgery instead of WFO is the inclusion criteria requiring normal eyes with minimal to no difference between subjective manifest refraction and topographic astigmatism calculated from the Topolyzer VARIO ${ }^{\circledR}$. When surgeons began treating complex patients, the question became the best way to determine refractive error using either topographic or measured manifest refraction. It was found that using topographically measured cylinder produced more desirable outcomes [6].

While these findings remain important, the uncertainty has led surgeons to use a combination of parameters including subjective manifest refraction, topographic astigmatism, and various other measurements to form a standardized approach for treatment. This led to the formation of Phorcides Analytic Engine Software which objectively determines the best treatment option by considering corneal astigmatism and elevation profile, topographic irregularities of higher order aberration, posterior corneal astigmatism, and lenticular astigmatism. They found no difference in the magnitude of residual refractive error between the Contoura ${ }^{\circledR}$ algorithm and Phorcides Analytic Engine ${ }^{\mathrm{TM}}$, however, there was a higher percentage of eyes with UCVA of 20/16 or better in the latter analytical software engine [7]. This software has further shown to decrease the amount of enhancement surgeries needed in post LASIK patients with rates down to $0.29 \%$ from earlier studies showing a rate of $0.7 \%$ with previous WFO treatment [8]. With the advances in analytic software for the planning of LASIK surgery, post-op visual outcomes have significantly improved while complications and the need for repeat laser surgery has decreased. During the 
COVID-19 pandemic, the safety of patients was jeopardized and the need for adjustments in preoperative and postoperative LASIK protocols were required while maintaining outcomes. In this study, we looked at the differences in visual outcomes for postoperative LASIK patients before and after the introduction of new regimens prior to and after surgery with reduced preoperative diagnostics and postoperative visits using telemedicine in ways never used before in our practice.

\section{Methods}

This study was a retrospective insignificant risk retrospective chart review and a comparative noninterventional study of postoperative outcomes of at least 12 months after uneventful LASIK using the Contoura ${ }^{\circledR}$ and Phorcides Analytic Engine ${ }^{\mathrm{TM}}$ algorithms on the WaveLight ${ }^{\circledR}$ laser and FS 200 femtosecond laser. Clinical results were obtained from one surgeon (K.G.S.) at 1 site from clinical charts. No protected health information was recorded.

Eligible eyes were those from patients who had previous LASIK with treatments in the approved range up to -9.0 diopters (D) of spherical equivalent myopia or myopia with astigmatism, with up to -8D of the spherical component and up to 3.0D of the astigmatic component at the spectacle plane. Both eyes were eligible for analysis. Surgical planning was based on either the manifest refraction (manifest group), Phorcides Analytic Engine ${ }^{\mathrm{TM}}$ (analytic group) or FDA topographic computed (TCAT group), with nomogram adjustments permitted in any group. Eyes with clinically significant pathology other than residual refractive error were excluded. Standard LASIK surgery was performed by one surgeon (K.G.S.) using the WaveLight ${ }^{\circledR}$ laser and FS 200 femtosecond laser under topical anesthesia with proparacaine hydrochloride 0.5\% topical (Akorn, Lake Forrest, IL, USA). Complications and enhancements were included in the analysis. Eyes treated for monovision or defocus were not included.

Manual and electronic data records were used to identify eyes that fit the inclusion and exclusion criteria. Deidentified data from the preoperative examination (age, sex, VA, and refraction), the treatment plan (keratometry and nomogram-adjusted treatment parameters), and the postoperative examination with up to 12 months postoperatively (VA and refraction) were collected; the source of the postoperative data (internal or from comanaging physicians) was also recorded. Where possible, any recorded enhancement in the first year after initial surgery was also recorded, although no eyes having undergone an enhancement before the recorded postoperative visit were included in the study pool. Out- comes of interest were the postoperative outcomes, complications, and differences between the groups. All statistical tests of hypotheses were based on a level of significance of $a=0.05$.

\section{Results}

This was a retrospective insignificant risk chart review and a comparative noninterventional study of 1,012 eyes of 506 patients treated with LASIK surgery before and after the COVID-19 pandemic at one site. The study group consisted of 676 eyes of 338 patients (Pre-COVID-19 group) and 336 eyes of 168 patients (PostCOVID-19 group). Mean preoperative refraction was -3.9+/- 0.85D for the Pre-COVID-19 group and -3.8+/-0.3D for the Post-COVID-19 group. The manifest refraction spherical equivalent ranged from 0 to -9.13D. The astigmatism ranged from 0 to 3.25D. Patients PreCOVID-19 received the following pre-op exam: diagnostic devices including VISX CustomVue ${ }^{\mathrm{TM}}$ Wavefront System (Johnson and Johnson, Santa Clara, CA, USA), Wavelight ${ }^{\circledR}$ Topolyzer $^{\circledR}$ VARIO (Alcon, Fort Worth, TX, USA), and Oculus Pentcam ${ }^{\circledR}$ Anterior Segment Tomography (Oculus, Arlington, WA, USA); refractions including wavefront, manifest and cycloplegic; physical exam including IOP Tonopen (Reichert, Depew, NY, USA) or iCare TA01i (Raleigh, NC, USA), slit lamp exam, and cycloplegic exam with cyclopentolate hydrochloride 1\% (Akorn, Lake Forrest, IL, USA) dilated fundoscopic exam. Patients Post-COVID-19 underwent a similar pre-op exam with the only main difference being all the exams performed in a patient dedicated room and no dilated exams (cycloplegic refraction, fundus exam) were performed. All tonometry in this group was non-contact with the iCare device, and the retinal examinations were done without dilation with the iCare DRS Plus ${ }^{\circledR}$ (Raleigh, NC, USA) as seen in figure 1. For Pre-COVID patients, their pre-op exam included in-patient appointments for diagnostics (CustomVue, Vario, and Pentacam images), refractions (wavefront, manifest, and cycloplegic), and exam (Slit lamp exam, intraocular pressure, and dilated fundus exam to include 20D and 90D exams) (Figure 2). For Pre-COVID-19 patients, their post- op exam included in-patient follow up appointments at one day, one week, one month, three months, and 12 months with the following measurements taken as seen in figure 3. For Post-Covid-19, there preoperative exam was the same as prior to Covid-19 without cycloplegia or dilation and non-contact intraocular pressure measurements as seen in figure 4. For Post-COVID-19 patients, their post- op exam included in-patient follow up at one day, three months, and 12 months and with telehealth visits at one week and one month using a questionnaire as seen in figure 5. The uncorrected visual acuity (UCVA) outcomes for patients in each group were measured and results are seen in 
figure 6. The postoperative day one vison was the primary endpoint to show rapidity of UCVA. As shown in the figure 7 below, post -COVID-19 patients had a slightly higher percentage of UCVA at $20 / 20: 100 \%$ vs $99 \%$ as well as UCVA of $20 / 15$ : $84 \%$ vs $81 \%$ respectively. Patients in the pre- COVID-19 group had higher rates of UCVA 20/10 and lower. Despite this numbers there were no statistically significant differences between the groups regarding outcomes between the 20/15 level and higher. Figure 6 shows the high positive correlation of UCVA between the two groups. Secondarily, it was found that the enhancement rates for the groups remained low at $0.29 \%$ with at least 1 year follow-up (Pre-COVID-19 2/676 and Post-COVID-19 1/336).

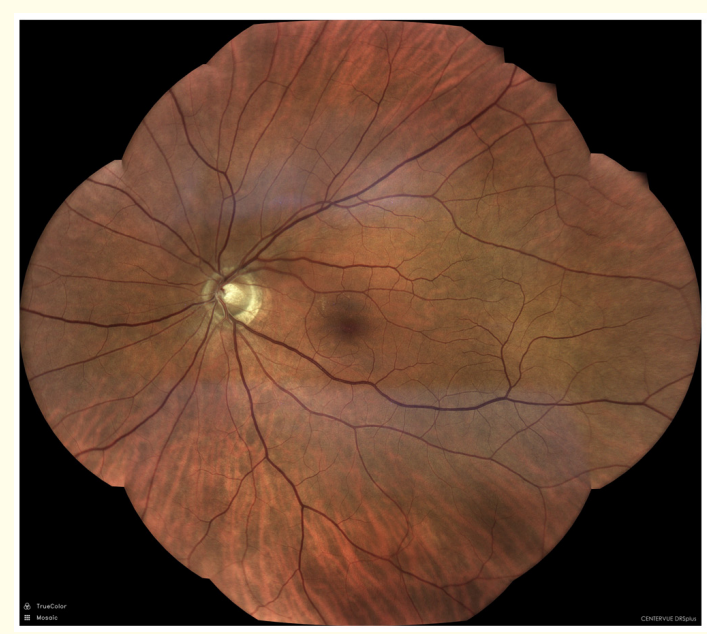

Figure 1: DRS Plus Retinal Exam.
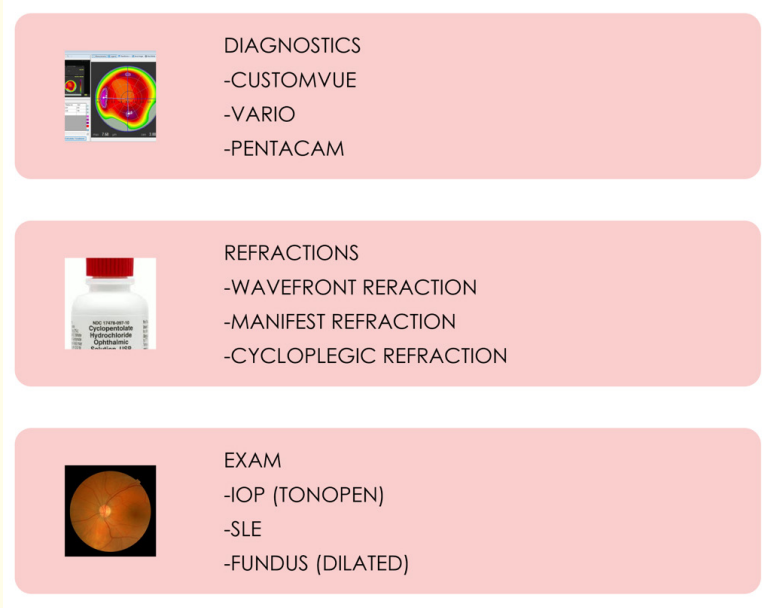

Figure 2: Pre-Covid Preoperative Exam.
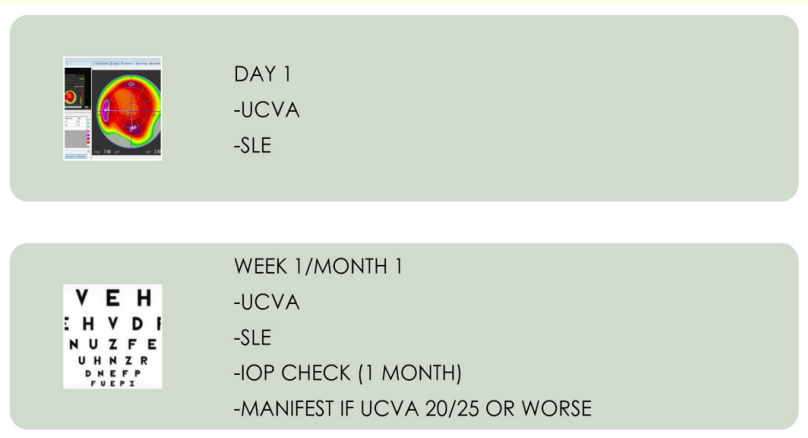

MONTH 3/MONTH 12
-IOP (TONOPEN)
-SLE
-MANIFEST REFRACTION (DATA POINT)
-FUNDUS (DILATED)

Figure 3: Pre-Covid Postoperative Exam.

DIAGNOSTICS (ALL IN ONE ROOM)
-CUSTOMVUE
-VARIO
-PENTACAM

REFRACTIONS (ALL IN PATIENT DEDICATED ROOM)
-WAVEFRONT RERACTION
-MANIFEST REFRACTION

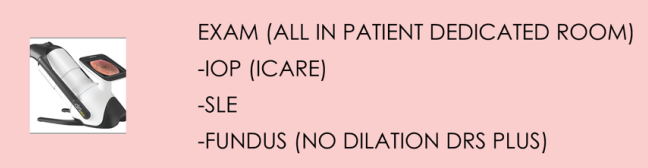

Figure 4: Post-Covid Preoperative Exam.

\section{Discussion}

Patient and staff safety are always at the forefront of any practice. Practitioners want to provide excellent outcomes while protecting their staff and patients from outside unforeseen sources. That is exactly what COVID-19 did to us. Other areas of ophthalmology were also affected with the COVID-19 outbreak. Several reports showed changes in cataract surgery and improved outcomes with reduced 

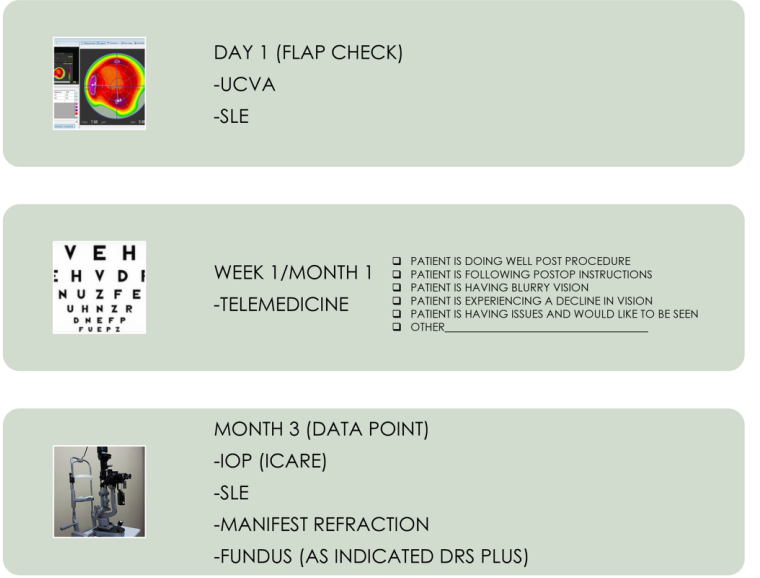

Figure 5: Post- Covid Postoperative Exam.

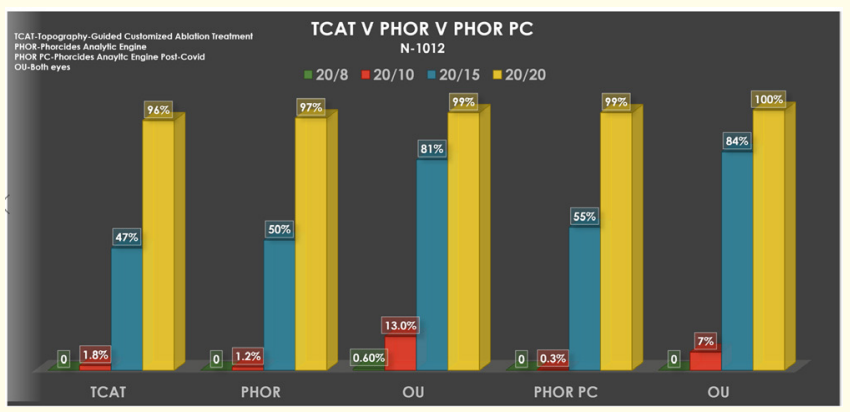

Figure 6: UCVA outcomes of Pre-COVID patient's vs Post-Operative Patients.

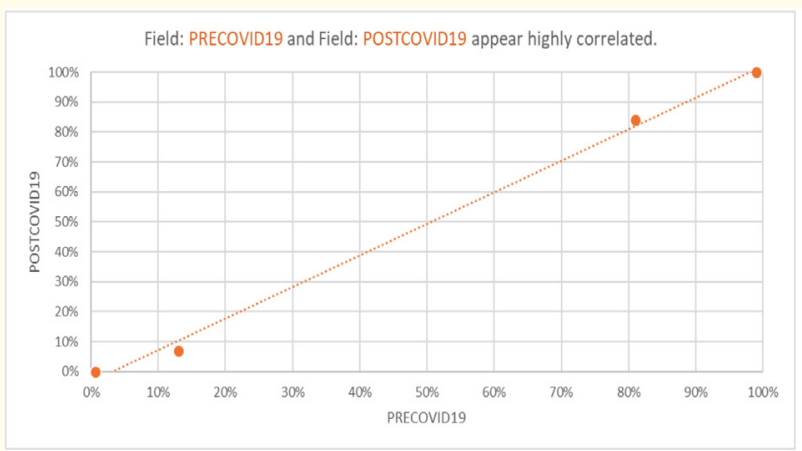

Figure 7: UCVA correlation of Pre-COVID patient's vs Post-Operative Patients. risk [9-11]. Due to the advances in computer software algorithms, surgeons are becoming more efficient at providing excellent surgical outcomes with low rates of complications and the need for enhancement surgery. With safety being even more important during the COVID-19 pandemic, the need for adjustments in post-op protocols were made to allow patients to be seen preoperatively, surgically treated, and then to be seen via telehealth from the safety of their homes post operatively. With no other changes, the visual outcomes and enhancement rates highly correlated. These findings provide insight into the possible adjustment of preoperative and postoperative management of LASIK patients that will allow a safer and more cost-efficient way to care for those after surgery. The most important statistic was no patients or staff during the treatment period developed COVID-19 to our knowledge.

\section{Conclusion}

The use of telehealth and a standardized questionnaire for follow up of post- op LASIK patients may provide a more cost efficient and safe protocol for the management of patients after laser surgery. Minor changes in preoperative work up and postoperative follow-up resulted in excellent and lasting outcomes.

\section{Bibliography}

1. Stewart Conor. Number of LASIK Surgeries in the United STATES 2020. Statista (2016).

2. Stulting RD., et al. "Results of topography-guided laser in situ keratomileusis custom ablation treatment with a refractive excimer laser". Journal of Cataract and Refractive Surgery 42.1 (2016): 11-18.

3. Mathew J and Stonecipher K. "Wavefront Optimized versus topography guided corneal ablations with the Wavelight ${ }^{\circledR}$ platform: A summary of visual outcomes". White Paper. 2020 Alcon Inc. 06/20 US-ALL-2000003.

4. Awwad ST and McCulley JP. "Wavefront-guided LASIK: recent developments and results". International Ophthalmology Clinic 46.3 (2006): 27-38.

5. Stonecipher K., et al. "Comparing wavefront-optimized, wavefront-guided and topography-guided laser vision correction: clinical outcomes using an objective decision tree". Current Opinion on Ophthalmology 29.4 (2018): 277-285.

6. Stulting RD., et al. "Topography-Guided Refractive Astigmatism Outcomes: Predictions Comparing Three Different Program- 
ming Methods". Clinical Ophthalmology 14 (2020): 1091-1100.

7. Lobanoff M., et al. "Clinical outcomes after topography-guided LASIK: comparing results based on a new topography analysis algorithm with those based on manifest refraction". Journal of Cataract and Refractive Surgery 46.6 (2020): 814-819.

8. Stonecipher MK and Stonecipher KG. "Influences on enhancement rates in laser vision correction". US Ophthalmic Review 9.2 (2016): 107-109.

9. Carr F., et al. "Restarting cataract surgery during the COVID-19 pandemic; a prospective study analyzing 30-day outcomes after elective cataract surgery in the United Kingdom". BMC Ophthalmology 21.1 (2021): 167.

10. Lin PF., et al. "Cataract service redesign in the post-COVID-19 era". British Journal of Ophthalmology 105.6 (2021): 745-750.

11. Bhalla JS., et al. "Immediate sequential bilateral cataract surgery and its relevance in COVID-19 era". Indian Journal of Ophthalmology 69.6 (2021): 1587-1591.

Volume 4 Issue 11 November 2021

(C) All rights are reserved by Karl Stonecipher., et al. 